\title{
The role of the skin in interoception: A neglected organ?
}

\author{
Laura Crucianelli ${ }^{+}$and H. Henrik Ehrsson \\ Department of Neuroscience, Karolinska Institutet, Stockholm
}

\begin{abstract}
In the past two decades, interoception has received increasing attention in the fields of psychology and cognitive science, as well as neuroscience and physiology. A plethora of studies adopted the perception of cardiac signals as a proxy for interoception. However, recent findings have cast doubt to the methodological and intrinsic validity of the tasks used thus far. Therefore, there is an ongoing effort to improve the existing cardiac interoceptive tasks and to identify novel channels to target the perception of the physiological state of the body. Amid such scientific abundancy, one could question whether the field has been partially neglecting one of our widest organs in terms of dimensions and functions, the skin. According to some views grounded on anatomical and physiological evidence, skin-mediated signals such as affective touch, pain, and temperature have been re-defined as interoceptive. Nevertheless, there is no agreement at this regard. Here, we discuss some of the anatomical, physiological, and experimental arguments supporting the scientific study of interoception by means of skin-mediated signals. We argue that more attention should be paid to the skin as a sensory organ that monitors the bodily physiological state, and further propose thermosensation as a particularly attractive model of skin-mediated interoception.
\end{abstract}

Keywords: Interoception, Skin, Thermosensation, Insula, Body awareness

\footnotetext{
${ }^{+}$Corresponding author:

Laura Crucianelli, PhD

Department of Neuroscience

Karolinska Institutet

Solnavägen 9, 17165 Solna

Stockholm, Sweden

Email: laura.crucianelli@ki.se
} 


\section{The concept and definitions of interoception}

A traditional classification for the perception of body related stimuli, relies on a distinction between exteroception (i.e., signals originating from outside the body), proprioception (i.e., signals about the position and movements of our limbs and body parts) (Proske \& Gandevia, 2012), and interoception (i.e., signals informing about the internal status of the body, Ceunen, Vlaeyen, \& Van Diest, 2016 for a review). The interplay between exteroceptive, proprioceptive, and interoceptive signals is important to update and maintain a coherent representation of our own body and for bodily awareness (Dijikerman, 2015; Crucianelli et al., 2018; Ehrsson, 2020). For decades psychological and neuroscience research has mainly focused on the perception of exteroceptive stimuli, such as visual, auditory signals, and discriminative touch (Bermúdez, Marcel \& Eilan, 1995) and, in the field of body representation research, most work has concentrated on proprioception and the integration of proprioceptive and exteroceptive signals (e.g., Collins et al 2005; Graziano et al 1999). More recently, there has been a substantial increase in attention and research on interoception (see also Khalsa et al., 2018).

There are several definitions of interoception and there is still no consensus on the one that should be adopted (see Chen et al., 2021; Quigley, Kanoski, Grill, Barrett \& Tsakiris, 2021 for recent reviews, and Table 1). Thus, there is a tendency to embrace the definition that suits the scientific approach or method used in each study. In its original definition, interoception was conceptualized as the body-tobrain axis of sensations concerning the state of the visceral body and its organs (Cameron, 2001; Sherrington, 1948, see Table 1), thus involving signals originating from within the body (e.g., cardiac, respiratory, and digestive functions). However, physiological and anatomical observations led to a redefinition and extension of interoception to encompass information about the physiological condition of the entire body, including also signals originating from many tissues of the body, such as the skin (e.g., temperature, itch, pleasure from gentle touch, and pain), and conveyed by specialized afferent pathways (Craig, 2002; Ceunen, Vlaeyen, \& Van Diest, 2016). In particular, the ideas highlighted in this paper are in line with the more inclusive definition provided by Craig, according to which interoception represents the perception of the physiological condition of the entire body at any given time (Craig, 2002, see Table 1). As such, the skin represents not only the boundaries of our body, but it also plays a fundamental role in homeostatic regulation by monitoring externally and internally generated signals about the body's physiological state of relevance for such self-regulation, and ultimately survival (Craig, 2003; Craig, 2008; Ehrsson et al., 2007; Björnsdotter, Morrison and Olausson, 2010; von Mohr and Fotopoulou, 2019; Burleson, \& Quigley, 2021; Crucianelli, Enmalm and Ehrsson, 2021).

The perception of temperature, pain, and gentle caress-like touch (which now is referred to as "affective touch") have traditionally been classified as submodalites of somatosensation (Sherrington, 1948), and thus often conceptualized as part of an exteroceptive modality that provides information about external objects and external events occurring on the skin. For example, when we explore objects with the digits, tactile and thermosensory impressions are seamlessly combined so that we can 
experience both the shape and roughness of the object together with its thermal properties; as such, a smooth spherical metal object feels cooler than the same shaped object made of wood, for example (e.g., Carnahan, Dubrowski \& Grierson, 2010). However, although these modalities are the results of stimulation on the body surface, thermosensation, affective touch, and cutaneous pain also carries information about the physiological state of the skin and the body in line with the mentioned redefinition of these as interoceptive submodalities (Craig, 2003, 2008), based on their affective, functional, physiological and and anatomical characteristics (Cabanac et al., 1972; Mower, 1976; Craig, 2002). Thus, this conceptualization emphasizes that these signals provide information about one's own body, i.e., it is you that feel cool or warm, in pain, or experience pleasure of an affective caress, so to highlight the affective-emotional dimensions of these sensory experiences. A critical argument for including signals from the skin into the concept of interoception comes from neuroanatomical considerations (Craig 2009; 2010). Noxious, thermal, and affective touch information that are signaled by special classes of receptors in the skin (see further below), reach the brain via different anatomical pathways through the spinal cord and thalamus than tactile and proprioceptive information (see further below). These signals target a different cortical area, the posterior insular cortex, which is crucial for interoception and processes visceral information. However, there is no consensus on the fact that certain skin signals should be defined as interoceptive.

Table 1. Summary of the most used definitions of interoception

\begin{tabular}{|c|c|}
\hline Citation & Definition of Interoception \\
\hline Sherrington, 1906 & Interoceptor: the sensory nerve receptors that reacted to stimuli originating within the body \\
\hline Sherrington, 1948 & $\begin{array}{l}\text { Body-to-brain axis of sensations concerning the state of the visceral body and its internal } \\
\text { organs }\end{array}$ \\
\hline Adam, 1998 & $\begin{array}{l}\text { Processing of information that is picked up by sensory receptors innervating the internal } \\
\text { organs and transmitted by ascending pathways of the autonomic nervous system }\end{array}$ \\
\hline Cameron, 2001 & $\begin{array}{l}\text { Visceral sensory nervous system impulses connecting body to brain to behaviour and } \\
\text { thought, with or without awareness }\end{array}$ \\
\hline Craig, 2002 & The sense of the physiological condition of the body at any given time \\
\hline Damasio, 2010 & The sensing of the organism's interior \\
\hline Critchley et al., 2004 & The sensing of the internal state of the body \\
\hline Dworkin, 2007 & Sensory visceral receptors that monitor the internal state of the body \\
\hline Barrett \& Simmons, 2015 & $\begin{array}{l}\text { The perception and integration of autonomic, hormonal, visceral and immunological } \\
\text { homeostatic signals that collectively describe the physiological state of the body }\end{array}$ \\
\hline $\begin{array}{l}\text { Ceunen, Vlaeyen, \& Van } \\
\text { Diest, } 2016\end{array}$ & $\begin{array}{l}\text { A multimodal integration not restricted to any sensory channel, not restricted to mere } \\
\text { sensations, but also relying on learned associations, memories, and emotions and integrating } \\
\text { these in the total experience which is the subjective representation of the body state }\end{array}$ \\
\hline Kahlsa et al., 2018 & $\begin{array}{l}\text { The overall process of how the nervous system senses, integrates, stores, and represents } \\
\text { information about the state of the inner body }\end{array}$ \\
\hline Oxford English Dictionary & $\begin{array}{l}\text { Any form of sensation arising from stimulation of interoceptors and conveying information } \\
\text { about the state of the internal organs and tissues, blood pressure, and the fluid, salt, and } \\
\text { sugar levels in the blood }\end{array}$ \\
\hline
\end{tabular}


Putting the issue of definitions aside, there is perhaps more agreement on the main function of interoception, which is subserving homeostatic regulation, i.e., the continuous neurobiological process that maintains a relative stability in the physiological condition of the body, despite internal and external changes (Cannon, 1929; Craig, 2008; Billman, 2020; Quigley et al., 2021), by supporting allostasis (i.e., the process of regulating peripheral systems in the body, Kleckner et al., 2017). Interoception is related to the autonomic nervous system and the generation of bodily (affective) feelings, informing the organism about its bodily needs (Craig, 2008, 2009; Damasio, 2010; Seth, 2013). Therefore, the impact of interoception is thought to extend beyond homeostatic regulation and also relates to the experience of emotions and the awareness of ourselves as feeling entities at any given time (Craig, 2009; Critchley et al., 2004; Damasio, 1994; Zaki, Davis \& Ochsner, 2012). Specifically, it has been proposed that the integration between interoceptive signals and exteroceptive information lies at the core of bodily awareness and self-consciousness (e.g., Simmons et al., 2013; Allen \& Tsakiris, 2018; Salvato et al., 2020; Park \& Blanke, 2019).

The purpose of this paper is to discuss some of the anatomical, physiological, and experimental arguments supporting the scientific study of interoception by means of skin-mediated signals. First, we will consider classic tasks to probe interoception through cardiac awareness and argue that the analysis of skin-based interoceptive signals provides a complementary and deeper understanding of interoception as a multifaceted construct. We will then also pay particular attention to thermosensation, which has been understudied in this regard, and propose that this sensory modality makes for a potential good model of skin-mediated interoception and review ongoing methodological advances in this direction.

\section{The problematic assessment of interoception}

Traditionally, the ability to perceive interoceptive signals has been quantified by asking participants to focus on their own heartbeats without touching their body but just by feeling the sensation of their heart beating (Schandry, 1981). In classic heartbeat counting tasks (HCT), participants are instructed to count their heartbeats during specific time windows (Dale \& Anderson, 1978; Schandry, 1981); an interoceptive accuracy index is then calculated using a formula that compares the numbers of actual and reported heartbeats. Given its relatively simple implementation and the quick procedure, this task became the main method used to quantify individual abilities in interoceptive accuracy (Ring \& Brener, 2018). However, the Schandry task has been criticised because it is not clear whether participants are counting their own heartbeats or rather keeping track of time and/or using previous knowledge to provide their best guess. Alternative methods to measure cardiac interoception are the heartbeat detection or discrimination tasks (HDT), whereby participants are asked to judge whether exteroceptive stimuli (e.g., auditory, or visual cues) are presented in synch or out of synch with their own heartbeats (e.g., Whitehead, Drescher, Heiman \& Blackwell, 1977; Katkin, Reed \& Deroo, 1983). Interestingly, the performances at these two types of tasks are unrelated, suggesting that they might assess different aspects of the perception of cardiac signals raising questions regarding how to best 
register accuracy (Ring \& Brener, 2018; Desmedt, Luminet \& Corneille, 2018). Other issues with such methods include evidence that the performance at heartbeat counting or detection tasks seem to be influenced by other factors such as prior knowledge, heart rates, beliefs, practice, and even experimental instructions (e.g., Ross \& Brener, 1981; Whitehead \& Drescher, 1980; Ring \& Brener, 1996; Ring et al., 2015; see Zamariola et al., 2018; Zimprich et al., 2020, Ainley et al., 2020; Corneille et al., 2020 for an extensive debate on the issues related to heartbeat tasks). Additionally, from the physiological point of view, the heartbeat signal itself can be problematic as it represents a multimodal, rather "noisy" signal, given the concurrent vascular and muscle contractions, which give rise to a cascade of other bodily signals (e.g., activation of tactile mechanoreceptors and volume of blood ejected at each heartbeat, Knapp-Kline, Ring, Emmerich \& Brener, 2021; Azzalini, Rebollo \& Tallon-Baudry, 2019). Thus, it is challenging to know whether participants are feeling the heartbeat signal per se, or whether they are using other bodily strategies to complete heartbeat detection or counting tasks (e.g., changes in respiration, tensing muscles, feeling pulsations in the fingertips etc, Ross \& Brener, 1981; Whitehead \& Drescher, 1980; Murphy et al., 2019). Furthermore, cardiovascular functions offer only one limited aspect of the broad palette of interoceptive signals. To overcome such limitations, the interoceptive field has witnessed a common effort to develop novel methods to quantify interoception, either by finding better ways to target the perception of cardiac signals (e.g., Larsson, Esposito, Critchley, Dienes \& Garfinkel, 2021; Legrand et al., 2021; Plans et al., 2021) or by focusing on other organs that provide interoceptive signals (see Zamairola et al., 2018; Ainley et al., 2020; Zimprich, Nusser \& Pollatos, 2020; Corneille et al., 2020 for a recent debate).

The maintenance of homeostasis is a sophisticated mechanism and does not rely solely on one basic function. Indeed, interoception extends beyond cardiac signals, and includes other signals originating from inside the body. Along this line, a few studies have attempted to investigate interoceptive abilities by focusing on other modalities, for example, gastric or stomach function (e.g., Whitehead \& Drescher, 1980; Herbert et al., 2012; Azzalini, Rebollo \& Tallon-Baudry, 2019), respiratory or breathing tasks (e.g., Garfinkel et al., 2016; Faull, Subramanian, Ezra \& Pattinson, 2019), and bladder functions (Griffiths, 2015; Ketai et al., 2016), but also thermal, nociceptive, and CT signals originating from the skin, which is the topic of the present work (Craig, 2002; 2009, see below for more details).

The question of whether interoception should be considered as a unitary concept or as a set of relatively independent submodules is an important one, both conceptually and from empirical perspectives. Although this is an area of ongoing research, some recent studies have found that interoception might be better conceptualised as a modular construct with relatively independent processing in parallel streams (Crucianelli, Enmalm and Ehrsson, 2021, see also Ferentzi et al., 2018, Garfinkel et al., 2016). For example, we recently investigated the relationships between cardiac interoception and several skin-mediated interoceptive modalities (i.e., pain, affective touch, and thermosensation in two tasks) and found that they are relatively independent (Crucianelli, Enmalm and 
Ehrsson, 2021). Thus, it is becoming increasing clear that to achieve a deep understanding of the concept of interoception, it should be quantified using a "battery of interoceptive tests" and attention should be paid to all channels, as each comes with specificities that uniquely contribute to the full picture of interoception.

\section{Probing interoception via the skin: Evidence from affective touch and cutaneous pain}

Probing interoception via external cutaneous stimuli can provide a more precise and controlled sensory signal as compared to internal stimulation (e.g., Craig, 2002; Björnsdotter, Morrison \& Olausson, 2010; Fotopoulou \& Tsakiris, 2017; Crucianelli et al., 2018; Crucianelli, Enmalm \& Ehrsson, 2021; Quigley et al., 2021). The skin, given its very nature, is a sensory organ extensively and directly exposed not only to the inside the body, but also to the external environment. As such, one of the reasons why skin signals might have been overlooked so far is the fact that they provide both interoceptive and exteroceptive sensory information, making it difficult to disentangle the two. Nevertheless, carefully designed and controlled experiments can allow us to manipulate only one component (i.e., the interoceptive one of interest), while keeping the other constant or absent (i.e., the exteroceptive ones). Given these premises, we argue that it is time to recognize the interoceptive nature of skin-mediated signals, in addition to the widely studied exteroceptive facet of touch.

The increasing focus on the study of affective touch and cutaneous pain has been partially motivated by the discovery of a specialized group of skin afferents, C Tactile (CT; Vallbo, Olausson \& Wessberg, 1999 for evidence in humans), that have been found mainly on the hairy skin of the body and has been proposed as one of the key afferent systems for affective touch (Löken, Wessberg, Morrison, McGlone \& Olausson, 2009; Morrison, Löken \& Olausson, 2010). In humans, CT afferents respond more vigorously to slow, caress-like touch, provided at a temperature typical of human skin (Vallbo et al. 1999, Wessberg et al. 2003, Löken et al. 2009, Ackerley et al. 2014); this specific type of tactile stimuli is more likely to be observed during spontaneous physical social interactions (Morrison et al., 2010; Morrison, 2016; Croy et al., 2016). Further support comes from neuroimaging studies that have shown that CT-signals are processed in key brain regions associated with interoception such as the insula and cingulate cortices (Morrison, 2016a; Morrison, 2016b for reviews; Björnsdotter et al. 2010; see further below). The characteristics and role of the CT system in for affiliative behaviours, affective touch, social bonding, and communication of emotions has been widely described and discussed, and fit with the view that CT processing and the associated subjective pleasant touch experiences should be considered as an interoceptive submodality (see Olausson, Lamarre, Backlund, Morin, Wallin et al., 2002; Löken et al., 2009; Kirsch et al., 2018; Morrison et al, 2010; Walker and McGlone, 2013, for reviews).

Similarly, pain can also be conceptualized as an interoceptive feeling and motivation (Craig, 2003). Pain has historically been seen both as a sensation and an emotion. The sensory dimension nociception - is related to activation of nociceptors and supports spatial localization and intensity 
encoding of the stimulus. The motivational-affective dimension - the subjective pain - arises centrally by further processing of nociceptive signals and integration of other sources of information (Basbaum \& Jessel, 2013). This latter motivational-affective dimension is involved in coding its valence (e.g., unpleasantness) and motivational relevance and has a more complex relationship to the original peripheral nociceptive signal. Thus, similar to affective touch, where one can distinguish between CTprocessing resulting from gentle mechanical stimulation of hairy skin and the resulting subjective pleasantness experience of affective touch, nociceptive processing and pain can also be distinguished in terms of sensory processing and later affective-emotional dimensions (e.g., Rainville et al. 1997; Hofbauer et al. 2001; Kulkarni et al. 2005; Auvray, Myin \& Spence, 2010). As in the case of hunger or thirst, pain represents a strong drive for action, which includes but it is not limited to changes in behavior (e.g., withdraw a body part), cognitive processes (e.g., try to focus the attention on something else, in the case of sustained pain) as well as social support (e.g., ask for help, Krahé, Springer, Weinman \& Fotopoulou, 2013). In line with this interoceptive-homeostatic view of pain, Morrison, Perini and Dunham (2013) proposed a Predictive Regulation and Action (PRA) model of acute pain processing, according to which the nervous system is organized to anticipate potential pain and to provide the motivation to take action to reduce the risk of tissue damage.

The feeling of cutaneous pleasure and pain are a common experience to all healthy human beings to various extents, but they are also subjective, as just described. These affective experiences are rather the result of elaborate and complex integration of various peripheral (i.e., activation of mechanoreceptors and nociceptors), multisensory (e.g. visual information), and contextual cues (e.g. social cues), and cognitive and emotional processes, which provides us with a rewarding, relaxing, and calming experience in the case of affective touch (Pawling, Cannon, McGlone and Walker, 2017), or modulates the perceived unpleasantness and distressing emotion, as in the case of pain (Farrar, 2010). As such, cutaneous pleasure and pain share more characteristics with interoceptive rather than exteroceptive modalities, given their homeostatic and affective nature (Craig, 2003). As we will discuss in the next section, we propose that thermosensation can be conceptualized in a similar way, arguing that it can be useful to distinguish between thermosensory processing and the sensory-discriminatory aspects of thermal stimulation, on the one hand, and the subjective affective feelings the processing of these signals also lead to, such as thermal comfort and discomfort.

\section{Thermosensation as an interoceptive modality}

"Sometimes when the sun appears in those shards you can touch, I'll stand motionless in the woods and let it fall on my cheek. I pretend it's my mother's hands on my face, the warmth so convincing that sometimes I'll even say a few words out loud..." 
Let us imagine a human being with no ability to monitor or regulate their own temperature: they would not be able to survive longer than a few hours (Sherwood \& Huber, 2010). The regulation of body temperature (thermoregulation) is one of the most vital concerns for many homeothermic animals, including humans (Craig, 2002; Tansey \& Johnson, 2015 for a review). Both breathing and thermoregulation contribute to the maintenance of homeostasis. While we have an organ just for the regulation of oxygen needs (i.e., lungs), there is not just one organ responsible for thermoregulation. Thus, the brain and the body are capable of activating almost immediate regulatory mechanisms against undesirable challenges to core body temperature (Proffitt, 2006; Davies et al., 2012; Filingeri et al., 2017). Involuntary physiological reactions may be involved, such as shivering or sweating, and these responses are activated at an early stage. Furthermore, voluntary temperature regulation takes place almost constantly (e.g., by changing clothing or the temperature of the room), driven by thermal stimuli which are perceived at the periphery, integrated at the central level, and lead to actions or reactions. Let us discuss two examples of this dual facet. On the one hand, the skin can help us to manage fever, that is an internal change in temperature due to an ongoing infection, in most cases. We all have experienced the situation of shivering and sweating when we are ill; this is the result of the skin helping the body thermoregulate its own temperature (Kurz, 2008). On the other hand, the skin constantly provides information about the external temperature, by activating the sensation of discomfort that we feel when we are too cold or warm, so that we are prompt to take actions against these thermal challenges. Thus, interoceptive responses to thermal stimuli can refer both to internally generated stimuli, but also in response to the application of thermosensory stimuli on the skin (Muzik and Diwadkar, 2016), both in the cases of heat and cold (Davis et al., 1998; Kwan et al., 2000).

Similarly, to the cardiac signals which are always present independently of the extent to which we are aware of them, we constantly perceive temperature via the skin. We are immersed in an external environment characterised by its own temperature, and the skin acts as an interface between the internal functions and the external environment. On a daily basis, we rely on signals mediated by the skin in order to regulate our homeostatic balance, and safety. Failures to regulate body temperature can have dramatic consequences for survival, as well as for physical and cognitive development (IJerman et al., 2015 for a review). Because such a narrow window of core body temperature is necessary for optimal functioning, the brain and the body do not only rely on bottom-up afferent signals to monitor bodily temperature but also have multiple means of predicting changes in temperature both in and outside the skin to maintain the temperature within the critical range more effectively. Indeed, the interoceptive nature of thermosensation can be investigated not only by focusing on peripheral perception, but also by considering descending predictions (see Morrison, Perini \& Dunham, 2013 for a similar approach in the perception of pain). For example, we do not need to touch an ice cube to know that it is cold: the mere vision of this object provides us with an embodied experience of 'what it would feel like' to touch or be touched by it. Thus, there might be anticipatory processes ('anticipation of thermosensation', Craig et al., 2000; Strigo, Matthews, \& Simmons, 2010, in line with the more general idea of 'interoceptive 
predictions', Barrett \& Simmons, 2015), taking place at the peripheral and central level that are activated even before any actual threat to thermoneutrality occurs. This process of anticipating thermal status and perceiving temperature generates an affective state of thermal comfort or discomfort, a feeling that signals its homeostatic role and is directly dependent on the body's needs to seek or avoid certain temperatures (Craig, 2002; Craig, 2003; Strigo \& Craig, 2016). The feeling of discomfort associated with being hot or cold is the way in which our body communicates that the maintenance of optimal body temperature is key for us to stay alive in changing environmental conditions as "naked apes". As such, the skin and thermosensation via the activation of voluntary and involuntary thermoregulatory processes, are able to guarantee the maintenance of our interoceptive balance via allostasis (e.g., Burleson and Quigley, 2021; IJzerman et al., 2015). Before turning to the issue of how to quantify thermonsensation as interoception in experimental behavioral studies, let us first consider the pathways from the skin to the brain in more detail.

\section{Specialized pathways from the skin to the brain}

"A person's own body, and above all its surface, is a place from which both external and internal perceptions may spring. It is seen like any other object, but to the touch it yields two kinds of sensations, one of which may be equivalent to an internal perception...The self is first and foremost a bodily self"

(Freud, S. The Ego and the ID. 1895, pp. 25-6)

The skin is our widest organ in terms of dimension and functions, and it wraps our entire body (Field, 2010; Montague, 1971; Serino and Haggard, 2010; Gallace and Spence, 2014). It is a very sophisticated system both in terms of internal structure and functions, and it is rich in diversity when it comes to specialized peripheral nerves systems activated in response to its stimulation (e.g., Corniani \& Saal, 2020). The versatile yet specialized nature of the skin and its afferents systems, plays an important role in the sense of touch as well as the skin-based interoceptive submodalites under discussion. Broadly speaking, the peripheral receptors in the skin can be mainly classified based on their dimension and conduction velocity. Namely, myelinated fibers (i.e., $A \beta$ ) are usually large and provide a fast response to stimulation; in contrast, small fibers provide a relative slower response to stimulation, and they can be unmyelinated fibers (i.e., C) or thinly myelinated (i.e., A $\delta$ ). The small, slower fibers are responsible for nociception, thermoception and affective touch (see Figure 1). The nociceptors, thermoceptors, and CT-tactile receptors are all free nerve endings, the most common nerve ending in the skin, sensitive to pressure (very light pressure in case of CT; extreme pressures in case of nociceptors), temperatures in different ranges (cold, cool, warm and heat in the case of thermoceptors; temperature extremes in case of nociceptors, $\left(>\sim 40^{\circ} \mathrm{C}-45^{\circ} \mathrm{C}\right.$ or $\left.<\sim 15^{\circ} \mathrm{C}\right)$ ), or chemicals signaling potential or actual tissue damage (Dubin \& Patapoutian, 2010; Jänig, 2018; Olausson et al., 2010). The sensory afferents conveyed by $\mathrm{C}$ - and $\mathrm{A} \delta$ fibers take a distinct pathway during development that reaches the spinal lamina I or solitary tract nucleus, which then connected to homeostatic/interoceptive nuclei of the thalamus (ventral medial 
posterior nucleus, VMPo). In contrast, the faster and larger $\mathrm{A} \beta$ fibers connect to the somatosensory/motor thalamic nuclei at a different stage during development (see Craig, 2015 for an overview). Critically, the neural signals carrying information about thermal, nociceptive, and pleasant touch stimuli from the VMPo then reach the contralateral posterior insular cortex (which is also the target of visceral inputs).

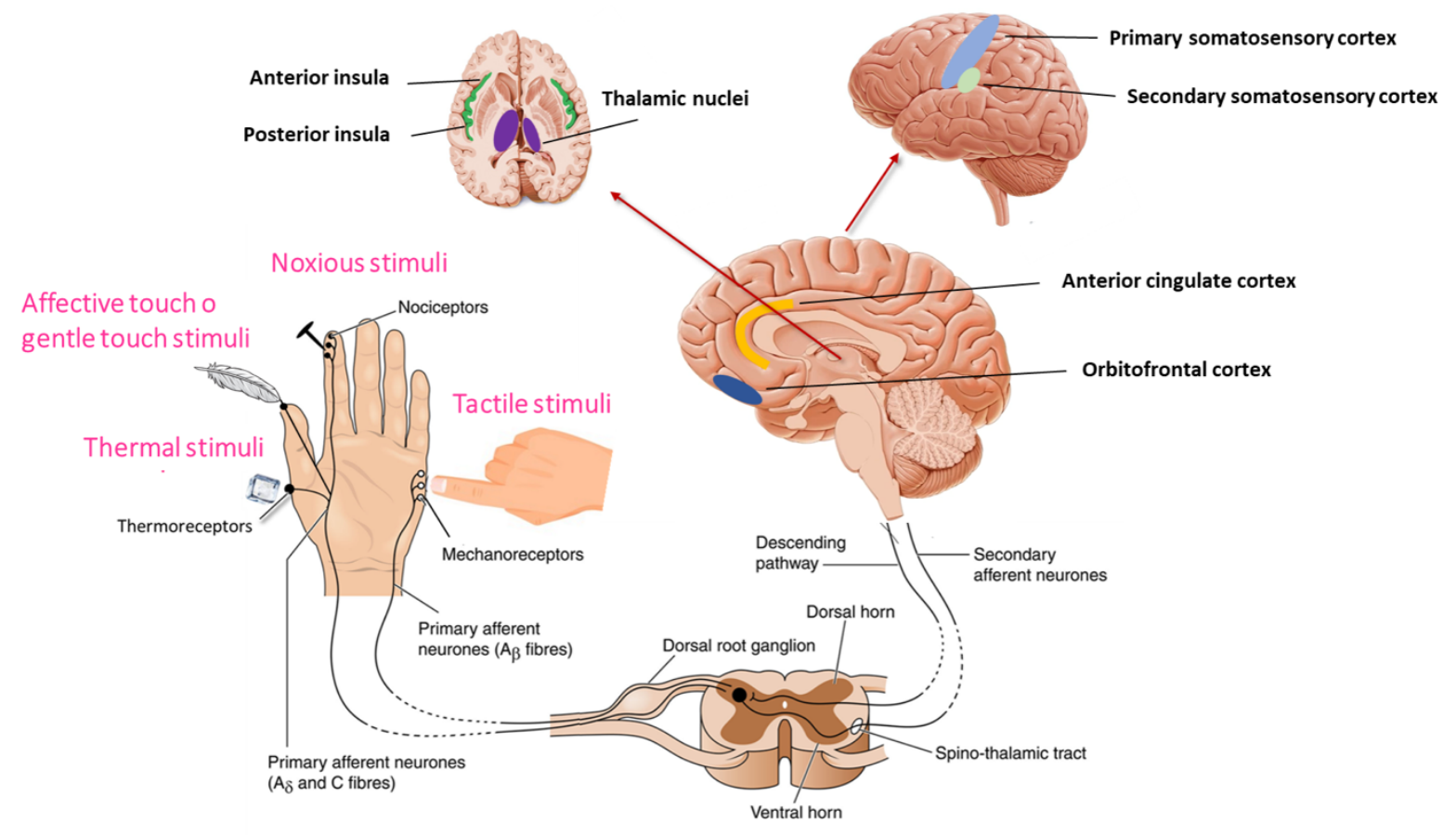

Figure 1. Pathways from the skin to the brain. For details see the text. (Figures adapted from kenhum.com and 123RF).

Via this pathway, the spinothalamocortical pathway, the thermal signals not only reach the posterior insular cortex (Craig et al., 2000; Hua et al., 2005), but also activate an autonomous thermoregulatory response in the preoptic area of the hypothalamus (Terrien et al., 2011; Nakamura and Morrison, 2008). From the thalamus and the posterior insula, the thermosensory signals are forwarded to multiple cortical areas, although relatively little is still known about these projections. However, from the posterior insula there are major connections to the anterior insular cortex where the information is further processed and integrated with other sources of information, including visceral information and exteroceptive signals. The anterior insular cortex has been proposed to assign affective meaning to the information arising from the original thermosensory stimulus (Craig, 2002; Muzik, Baajour, Bressler \& Diwadkar, 2020; Satinoff, 1978; Diwadkar et al., 2014; Craig et al., 2000; Craig, 2018; Evrard, 2019). In parallel, thermal signals also reach the parietal cortex and the primary somatosensory cortex, possibly subserving sensory-discrimination and stimulus localization (Solinski \& Hoon, 2019; Gallace et al., 2014), as well as the anterior cingulate cortex and the orbitofrontal cortex (OFC) that might support more motivational dimensions of the thermal experience, including subjective feelings associated with 
the pleasantness or the unpleasantness (Rolls, 2010), which in turn will motivate behaviour. The cortical responses associated with thermosensory stimulation have also been described as a hierarchically organized thermoregulatory network that is able to distinguish between cold and warm stimuli (Muzik and Diwadkar, 2016). How the processing of thermoceptive signals turn into affective-interoceptive feelings of thermal comfort and discomfort more exactly is not clear (e.g., Oi et al., 2017), but we speculate that it is likely to involve an interplay of insular, cingulate, and orbitofrontal areas, and this is an important question for future research.

Gentle touch stimuli delivered at CT-optimal speed activates the posterior insular cortex in human subjects (Björnsdotter et al., 2009). Activations of anterior insula, cingulate cortex, and orbitofrontal cortex are also seen during affective touch (McGlone et al., 2012; Rolls et al., 2003; Case et al., 2016). Although such gentle stroking also activates the classical somatosensory areas, the primary somatosensory cortex and the secondary somatosensory cortex (due to co- stimulation of larger $\mathrm{A} \beta$ fibers), a meta-analysis suggests that posterior insula is more likely to be activated for affective touch, and primary somatosensory cortices (SI) is more likely to be activated for discriminative touch (Morrison, 2016). Moreover, pleasantness ratings correlate more than intensity ratings in activity in the cingulate cortex, while S1 activity only correlated with intensity ratings, highlighting the cingular contribution to the affective dimension of the gentle touch (Case et al 2016). Correlation between neural activity and touch pleasantness have also been reported in the OFC (McCabe et al. 2008), although such correlations are apparently typically not seen for the posterior insula (Case et al 2016; but see Kress et al., 2011). However, lesion of the insular cortex following right-hemisphere stroke, disrupts the perception of tactile pleasantness rather than tactile intensity (Kirsch et al., 2020). Thus, pleasantness from gentle touch might arise as a consequence of further processing of CT-signals originating from the posterior insular cortex in the anterior insula, cingulate cortex, orbitofrontal cortex through integration with other sources of information.

Nociceptive processing and the subjective experience of pain are associated with the activation of a network of brain regions, including primary and secondary somatosensory cortex, the anterior cingulate cortex, and the insular cortices, (e.g., Duerden \& Albanese, 2013 and Jensen et al., 2016 for a meta-analysis). Famously, there is no "primary nociceptive cortex", rather nociceptive signals are processes in several areas including the insular cortex and the primary somatosensory cortex (area $3 \mathrm{a}$ ). The subjective experience of pain is thought to arise as a consequence of interactions (Kastrati et al., 2022) between brain regions involved in nociceptive processing (Jensen et al., 2016) and regions supporting cognition and emotion (Geuter et al., 2020). Of particular interest in this context is processing of nociceptive signals in the midcingulate cortex and bilateral posterior insula, which are not only often seen during nociceptive stimulation but is also under significant genetic influence (Kastrati et al., 2022), in line with an evolutionally conserved system, which one would expect for a life-sustaining critical interoceptive function. 
The similarities in the organization principles of the anatomical pathways and central processing architecture for thermosensation, affective touch, and nociception/pain are one of the key arguments for the proposed re-definition of such modalities as homeostatically-relevant and interoceptive because they all carry not only discriminative perceptual qualities but also emotional feelings about the body's physiological state (Craig, 2003).

\section{Thermosensation as skin-based interoception: novel experimental directions}

Among the skin-based interoceptive submodalities, thermosensation offers numerous advantages from an experimental and methodological point of view. Stimulation can be easily experimentally controlled, in the sense that we can systematically manipulate the temperature we deliver on the skin with high precision (e.g., $\pm 0.1-0.2^{\circ} \mathrm{C}$, Somedic SenseLab AB, Hörby, Sweden), while recording both the subjective perception (e.g., via a rating scale or detection/discrimination tasks), the objective physiological state of the skin (i.e., temperature), and the physiological reaction (e.g., change in body temperature) to such stimulation (Crucianelli, Enmalm \& Ehrsson, 2021; Radziun, Crucianelli \& Ehrsson, 2021). Moreover, it is possible to deliver very selective activation of thermoreceptors in the skin, which can be done with contactless radiant stimulation (heat lamps), dry ice kept at a close distance from the skin, or by laser stimulation (e.g. Nd: YAP Laser - Stimul 1340, Stimul 1340 Neurolas, Deka, Calenzano Italy). Such stimulation can also be given without an external object touching the skin, which eliminates the potential binding of the thermal experiences to the external object, thus ensuring that the thermal sensations are perceived to be originating from one's own body. It is also possible to present thermal stimuli that, to various degree, "threatens" thermoneutrality by presenting stimuli that are cooler or warmer than normal skin temperature to probe the resulting feelings of thermal comfort and discomfort. The latter is an advantage compared to nociceptive and CT-optimal tactile stimulation as feelings of pleasure and pain are "one-directional", either triggered or absent, rather than changing around a homeostatic target level. Compared to affective touch, it is also easier to selectively activate thermoreceptors than CT-afferents (with radiant stimulation or laser). Moreover, in contrast to affective touch or pain, temperature does not necessarily have a strong affective component when manipulated within the innocuous range (cool to warm perception), which is an advantage in experimental studies as it is easier to match conditions and it raises fewer ethical issues than when administering pain. Studying interoception via thermosensation is less invasive since it can be prompt externally, if compared to other methods used thus far to investigate interoception, such as gastric or bladder functions.

The tasks used to study thermosensation over the last century have focused on sensorydiscriminative nature of this sense in line with most work on somatosensation. We suggest that, by applying the interoceptive principles discussed above, one can design a new generation of thermosensory tasks that more directly probe the affective aspects of thermosensation, such as subjective feeling of thermal comfort and discomfort. 
In our lab we currently work on several such new tasks, and one is named the thermal matching task (see Figure 2) (Crucianelli, Enmalm \& Ehrsson, 2021; Radziun, Crucianelli \& Ehrsson, 2021), which is based on concepts from the affective touch literature and thermosensation as interoception. In this task, participants are asked to recognise a previously perceived moving thermal stimulus applied to the skin at CT-optimal velocity when presented among other warmer or cooler stimuli. The temperatures are within the range of thermoneutrality $\left(30-34^{\circ} \mathrm{C}\right)$ and we register how accurate participants are in detecting thermal stimuli within that range, and we also explore differences between hairy (rich in CT afferents) and non-hairy skin (where CTs are sparse) since CT afferents are tuned to respond optimally to typical skin temperature (Ackerley et al 2014). The results reveal greater thermosensory sensitivity on hairy skin in line with the idea that temperature perception around thermoneutrality on hairy skin might be based on C-fibres such as classic cold and warm thermal afferents (i.e., $\mathrm{C}$ and $\mathrm{A} \delta$ ), and potentially also CT signals, which might work in concert with cold and warm receptors by detecting and signal deviations from their optimal temperature sensitivity (i.e., $32^{\circ} \mathrm{C}$ ) (Craig, 2009; Björnsdotter, Morrison \& Olausson, 2010; Morrison, 2016; Burleson \& Quigley, 2021). This task can be easily extended to include judgements of thermal comfort and the perceived pleasantness of touch, and by varying the velocity, temperatures, and skin types stimulated to tease apart the relationships between thermal discrimination, thermal comfort, and tactile pleasantness.

\section{Thermal Matching Task \\ Crucianelli, Enmalm, \& Ehrsson, 2021}

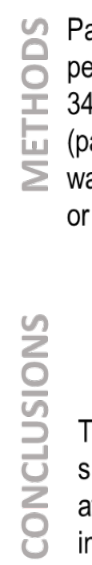

Participants have to match a previously perceived moving thermal stimulus (30$34^{\circ} \mathrm{C}$ ) on hairy (forearm) and non-hairy (palm) skin to a range of colder or warmer stimuli, presented in increasing or decreasing order.

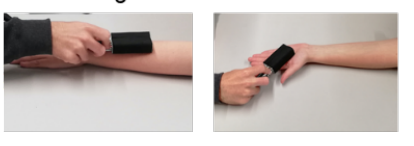
interoception.
The Thermal Matching Task shows more accurate perception of dynamic temperature on hairy compared to non-hairy skin overall, particularly when temperature was decreasing
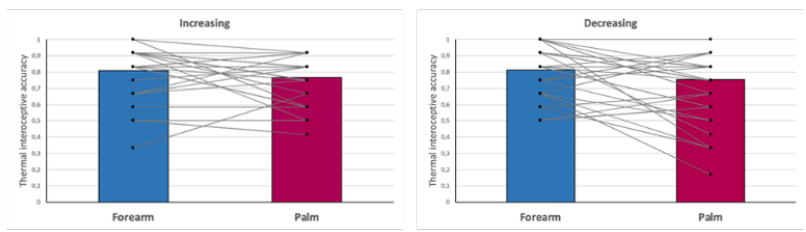

The perception of temperature on hairy skin might relate to, at least in part, the sensation of homeostatic internal signals from our body mediated by $\mathrm{C}$-fibres such as classic cold and warm thermal afferents, and potentially CT afferents. Thus, probing thermosensation might offer a promising avenue to investigate skin-mediated

Figure 2. Summary of the methods, results, and conclusions of the Thermal Matching Task, fully described in Crucianelli, Enmalm \& Ehrsson (2021). In line with classic approaches in the cardiac interoceptive field (e.g., Schandry, 1989), we applied a formula which allowed us to conveniently obtain a number between 0 and 1 , where 0 indicates lower ability to detect changes from thermoneutrality (worst performance at the task) and 1 indicates higher such ability (better performance at the task). This index of thermal interoceptive ability can then be compared to similar indexes calculated for other interoceptive tasks in a "battery" of tests to probe different interoceptive submodalites.

\section{Skin-based interoception in social behaviors and bodily awareness}


Because skin-based interoception provides information about how the skin feels at any given movement, it may also subserve functions in bodily awareness and social interactions. A few studies have suggested a role of CT-signals and tactile pleasantness to the sense of the body as one's own (body ownership; Crucianelli et al., 2013, 2018; Lloyd et al., 2013; van Stralen et al., 2014). Body ownership is a multisensory construct whereby different streams of sensory signals are being combined into a coherent multisensory representation of one's own body (Ehrsson et al 2020). Although most previous studies have focused on the integration of visual, tactile, and proprioceptive signals, we know that the sense of body ownership is closely linked to functions of defending the body and emotional defense reactions (Ehrsson et al., 2007; Graziano \& Cooke, 2005), which thus indicate an important role for interoception (Tsakiris, 2017). Ongoing studies has begun to use selective stimulation of thermoreceptors and nociceptors using contactless radial stimulation and laser stimulation to better understand the precise contribution of inputs from thin unmyelinated C-fibers to body ownership. Hence by studying skin-based interoception we can obtain a better understanding about the interplay between exteroception, proprioception, and interoception for the sense of body ownership.

Skin-based interoception support inter-personal behavioral and social cognition, and probing interoception via thermosensation might offer a particularly intriguing opportunity to study the link between social connection and bodily signals (Arnold, Winkielman \& Dobkins, 2019). According to some views, the way in which we learn to read, interpret, and respond to thermal signals is also via social tactile interactions with our caregivers (e.g., Fotopoulou \& Tsakiris, 2017; Ciaunica \& Fotopolou, 2017; Ciaunica \& Crucianelli, 2018). At birth, we do not have the means to act upon our interoceptive needs, such as food intake and behavioural thermoregulation (i.e., cover or uncover us up) and we rely on others to take care of our survival. Thus, social touch is a fundamental tool to cope with stressors and challenges via physiological regulation of our bodily states (Morrison, 2016; Fotopoulou \& Tsakiris, 2017). Social physical contact and proximity such as when hugging and snuggling, are also fundamental processes of social thermoregulation, one of the most economical and efficient way to keep our body at a good temperature (Morrison, 2016; IJerman et al., 2015). Through social embodied interaction with others, we can guarantee our most optimal social functioning in terms of emotion, thermoregulation, and ultimately survival as a species (IJerman et al., 2015 for a review).

\section{Concluding Remarks}

Here, we integrated some of the physiological, behavioral, and neuroanatomical evidence in support of the interoceptive nature of some skin-mediated signals. In particular, we have highlighted the strengths and advantaged of studying interoception by focusing on the skin, given its dual nature of being exposed to the internal environment of our body and to the external world. We suggested that thermosensation - in addition to affective touch and cutaneous pain - could be considered as a valid model of skin-mediated interoception and argued that experimental studies that allow to control for or eliminate the exteroceptive component of thermosensation, can allow to target the interoceptive facet of 
this modality. Moreover, investigating interoception via skin stimulation can provide a unique insight into bodily awareness as well as a better understanding of clinical conditions characterized by disorders of thermoregulation, anhedonia (i.e., the inability to experience pleasure), and chronic pain (i.e., persistent experience of pain past normal healing time), to name a few. Thus, the skin is the sensory organ that can afford us promising opportunities to improve the scientific study and understanding of interoception, and its clinical and experimental applications.

\section{Acknowledgments}

This work was supported by the European Research Council under the European Union's horizon 2020 research and innovation programme (SELF-UNITY) to H. Henrik Ehrsson. Laura Crucianelli was supported by the Marie Skłodowska-Curie Intra-European Individual Fellowship (HOMEOTHERMIC SELF). 


\section{References}

Ádám, G. (1998). Conceptual Preliminaries. In Visceral Perception (pp. 31-35). Springer, Boston, MA.

Ackerley, R., Wasling, H. B., Liljencrantz, J., Olausson, H., Johnson, R. D., \& Wessberg, J. (2014). Human Ctactile afferents are tuned to the temperature of a skin-stroking caress. Journal of Neuroscience, 34(8), 2879-2883.

Ainley, V., Tsakiris, M., Pollatos, O., Schulz, A., \& Herbert, B. M. (2020). Comment on "Zamariola et al.(2018), Interoceptive Accuracy Scores are Problematic: Evidence from Simple Bivariate Correlations"-The empirical data base, the conceptual reasoning and the analysis behind this statement are misconceived and do not support the authors' conclusions. Biological psychology, 152, 107870.

Allen, M., \& Tsakiris, M. (2018). The body as first prior: Interoceptive predictive processing and the primacy. The interoceptive mind: From homeostasis to awareness, 27.

Arnold, A. J., Winkielman, P., \& Dobkins, K. (2019). Interoception and social connection. Frontiers in psychology, 10, 2589.

Auvray, M., Myin, E., \& Spence, C. (2010). The sensory-discriminative and affective-motivational aspects of pain. Neuroscience \& Biobehavioral Reviews, 34(2), 214-223.

Azzalini, D., Rebollo, I., \& Tallon-Baudry, C. (2019). Visceral signals shape brain dynamics and cognition. Trends in Cognitive Sciences, 23(6), 488-509.

Barrett, L. F., \& Simmons, W. K. (2015). Interoceptive predictions in the brain. Nature reviews neuroscience, 16(7), 419-429.

Basbaum, A.I., \& Jessell, T.M. (2013). Pain. In: Kandel ER, Schwartz JH, Jessell TM, (Eds). Principles of neural science. McGraw-Hill, International Edition

Bermúdez, J. L., Marcel, A. J., \& Eilan, N. (Eds.). (1995). The body and the self. The MIT Press.

Billman, G. E. (2020). Homeostasis: the underappreciated and far too often ignored central organizing principle of physiology. Frontiers in physiology, 11, 200.

Björnsdotter, M., Löken, L., Olausson, H., Vallbo, Å., \& Wessberg, J. (2009). Somatotopic organization of gentle touch processing in the posterior insular cortex. Journal of Neuroscience, 29(29), 9314-9320.

Björnsdotter, M., Morrison, I., \& Olausson, H. (2010). Feeling good: on the role of C fiber mediated touch in interoception. Experimental brain research, 207(3-4), 149-155.

Burleson, M. H., \& Quigley, K. S. (2021). Social interoception and social allostasis through touch: legacy of the somatovisceral afference model of emotion. Social neuroscience, 16(1), 92-102.

Cabanac, M., Massonnet, B., \& Belaiche, R. (1972). Preferred skin temperature as a function of internal and mean skin temperature. Journal of applied physiology, 33(6), 699-703.

Cameron, O. G. (2001). Visceral sensory neuroscience: Interoception. Oxford University Press.

Cannon, W. B. (1929). Organization for physiological homeostasis. Physiological reviews, 9(3), 399-431.

Carnahan, H., Dubrowski, A., \& Grierson, L. E. (2010). Temperature influences both haptic perception and force production when grasping. International Journal of Industrial Ergonomics, 40(1), 55-58.

Case, L. K., Laubacher, C. M., Olausson, H., Wang, B., Spagnolo, P. A., \& Bushnell, M. C. (2016). Encoding of touch intensity but not pleasantness in human primary somatosensory cortex. Journal of Neuroscience, 36(21), 5850-5860.

Ceunen, E., Vlaeyen, J. W., \& Van Diest, I. (2016). On the origin of interoception. Frontiers in psychology, 7, 743.

Chen, W. G., Schloesser, D., Arensdorf, A. M., Simmons, J. M., Cui, C., Valentino, R., ... \& Langevin, H. M. (2021). The emerging science of interoception: sensing, integrating, interpreting, and regulating signals within the self. Trends in neurosciences, 44(1), 3-16.

Ciaunica, A., \& Fotopoulou, A. (2017). The touched self: Psychological and philosophical perspectives on proximal intersubjectivity and the self. In Durt, C., Fuchs, T. \& Tewes, C. Embodiment, enaction, and Culture investigating the constitution of the shared world, 173-192.

Ciaunica, A., \& Crucianelli, L. (2019). Minimal self-awareness from within: A developmental perspective. Journal of Consciousness Studies, 26(3-4), 207-226.

Collins, D. F., Refshauge, K. M., Todd, G., \& Gandevia, S. C. (2005). Cutaneous receptors contribute to kinesthesia at the index finger, elbow, and knee. Journal of neurophysiology, 94(3), 1699-1706. 
Corneille, O., Desmedt, O., Zamariola, G., Luminet, O., \& Maurage, P. (2020). A heartfelt response to Zimprich et al. (2020), and Ainley et al. (2020)'s commentaries: Acknowledging issues with the HCT would benefit interoception research. Biological Psychology, 152, 107869.

Corniani, G., \& Saal, H. P. (2020). Tactile innervation densities across the whole body. Journal of Neurophysiology, 124(4), 1229-1240.

Craig, A. D., Chen, K., Bandy, D., \& Reiman, E. M. (2000). Thermosensory activation of insular cortex. Nature Neuroscience, 3(2), 184-190. doi:10.1038/72131

Craig, A. D. (2002). How do you feel? Interoception: the sense of the physiological condition of the body. Nature Reviews Neuroscience, 3. 655-666.

Craig, A. D. (2003). A new view of pain as a homeostatic emotion. Trends in neurosciences, 26(6), 303-307.

Craig, A. D. (2008). Interoception and emotion: a neuroanatomical perspective. Handbook of emotions, 3(602), $272-88$.

Craig, A. D. (2009). How do you feel-now? The anterior insula and human awareness. Nature reviews neuroscience, 10(1).

Craig, A. D. (2015). How do you feel?. Princeton University Press.

Critchley, H. D., Weins, S., Rohstein, P., Öhman, A., \& Dolan, R. J. (2004). Neural systems supporting interoceptive awareness. Nature Neuroscience, 7(2), 189-195. doi:10.1038/nn1176

Croy, I., Luong, A., Triscoli, C., Hofmann, E., Olausson, H., \& Sailer, U. (2016). Interpersonal stroking touch is targeted to C tactile afferent activation. Behavioural Brain Research, 297, 37-40.

Crucianelli, L., Metcalf, N. K., Fotopoulou, A. K., \& Jenkinson, P. M. (2013). Bodily pleasure matters: velocity of touch modulates body ownership during the rubber hand illusion. Frontiers in psychology, 4, 703.

Crucianelli, L., Krahé, C., Jenkinson, P. M., \& Fotopoulou, A. (2018). Interoceptive ingredients of body ownership: Affective touch and cardiac awareness in the rubber hand illusion. Cortex, 104, 180-192. doi: 10.1016/j.cortex.2017.04.018.

Crucianelli, L., Enmalm, A., \& Ehrsson, H. H. (2021). Thermosensation as a novel method to probe interoception and its relationship with other interoceptive modalities. bioRxiv.

Dale, A., \& Anderson, D. (1978). Information variables in voluntary control and classical conditioning of heart rate: Field dependence and heart-rate perception. Perceptual and Motor Skills, 47(1), 79-85.

Damasio, A. R. (1994). Descartes' error: Emotion, rationality and the human brain.

Damasio, A. (2010). Self Comes to Mind: Constructing the Conscious Brain. Pantheon.

Davies, N. B., Krebs, J. R., and West, S. A. (2012). An Introduction to Behavioural Ecology. New York: John Wiley \& Sons

Davis, K. D., Kwan, C. L., Crawley, A. P., \& Mikulis, D. J. (1998). Functional MRI study of thalamic and cortical activations evoked by cutaneous heat, cold, and tactile stimuli. Journal of Neurophysiology, 80(3), 15331546.

Desmedt, O., Luminet, O., \& Corneille, O. (2018). The heartbeat counting task largely involves non-interoceptive processes: Evidence from both the original and an adapted counting task. Biological Psychology, 138, 185188.

Dubin, A. E., \& Patapoutian, A. (2010). Nociceptors: the sensors of the pain pathway. The Journal of clinical investigation, 120(11), 3760-3772.

Duerden, E. G., \& Albanese, M. C. (2013). Localization of pain-related brain activation: A meta-analysis of neuroimaging data. Human brain mapping, 34(1), 109-149.

Dworkin, B. R. (2007). Interoception. In J. T. Cacioppo, L. G. Tassinary, \& G. G. Berntson (Eds.), Handbook of psychophysiology (p. 482-506). Cambridge University Press

Ehrsson, H. H., Wiech, K., Weiskopf, N., Dolan, R. J., \& Passingham, R. E. (2007). Threatening a rubber hand that you feel is yours elicits a cortical anxiety response. Proceedings of the National Academy of Sciences, 104(23), 9828-9833.

Ehrsson, H. H. (2020). Multisensory processes in body ownership. In: Sathian, K., Ramachandran, V.S., eds. Multisensory Perception: From Laboratory to Clinic (pp. 179-200), Academic Press: Elsevier.

Evrard, H. C. (2019). The organization of the primate insular cortex. Frontiers in neuroanatomy, 13, 43.

Farrar, J. T. (2010). Advances in clinical research methodology for pain clinical trials. Nature medicine, 16(11), 1284 
Faull, O. K., Subramanian, H. H., Ezra, M., \& Pattinson, K. T. (2019). The midbrain periaqueductal gray as an integrative and interoceptive neural structure for breathing. Neuroscience \& Biobehavioral Reviews, 98, 135-144.

Ferentzi, E., Bogdány, T., Szabolcs, Z., Csala, B., Horváth, Á., \& Köteles, F. (2018). Multichannel investigation of interoception: Sensitivity is not a generalizable feature. Frontiers in human neuroscience, 12, 223.

Field, T. (2010). Touch for socioemotional and physical well-being: A review. Developmental review, 30(4), 367383.

Filingeri, D., Morris, N. B., \& Jay, O. (2017). Warm hands, cold heart: progressive whole-body cooling increases warm thermosensitivity of human hands and feet in a dose-dependent fashion. Experimental physiology, 102(1), 100-112.

Fotopoulou, A., \& Tsakiris, M. (2017). Mentalizing homeostasis: The social origins of interoceptive inference. Neuropsychoanalysis, 19(1), 3-28.

Gallace, A., \& Spence, C. (2014). In touch with the future: The sense of touch from cognitive neuroscience to virtual reality. OUP Oxford.

Gallace, A., Soravia, G., Cattaneo, Z., Moseley, G. L., \& Vallar, G. (2014). Temporary interference over the posterior parietal cortices disrupts thermoregulatory control in humans. PLoS One, 9(3), e88209.

Garfinkel, S. N., Manassei, M. F., Hamilton-Fletcher, G., In den Bosch, Y., Critchley, H. D., \& Engels, M. (2016). Interoceptive dimensions across cardiac and respiratory axes. Philosophical Transactions of the Royal Society B: Biological Sciences, 371(1708), 20160014.

Geuter, S., Reynolds Losin, E. A., Roy, M., Atlas, L. Y., Schmidt, L., Krishnan, A., ... \& Lindquist, M. A. (2020). Multiple brain networks mediating stimulus-pain relationships in humans. Cerebral Cortex, 30(7), 42044219.

Graziano, M. S. (1999). Where is my arm? The relative role of vision and proprioception in the neuronal representation of limb position. Proceedings of the National Academy of Sciences, 96(18), 10418-10421.

Graziano, M. S., \& Cooke, D. F. (2006). Parieto-frontal interactions, personal space, and defensive behavior. Neuropsychologia, 44(6), 845-859.

Griffiths, D. (2015). Neural control of micturition in humans: a working model. Nature reviews Urology, 12(12), 695-705.

Herbert, B. M., Muth, E. R., Pollatos, O., \& Herbert, C. (2012). Interoception across modalities: on the relationship between cardiac awareness and the sensitivity for gastric functions. PloS one, 7(5), e36646.

Hofbauer, R. K., Rainville, P., Duncan, G. H., \& Bushnell, M. C. (2001). Cortical representation of the sensory dimension of pain. Journal of neurophysiology, 86(1), 402-411.

Hua, L. H., Strigo, I. A., Baxter, L. C., Johnson, S. C., \& Craig, A. D. (2005). Anteroposterior somatotopy of innocuous cooling activation focus in human dorsal posterior insular cortex. American Journal of Physiology-Regulatory, Integrative and Comparative Physiology, 289(2), R319-R325.

IJzerman, H., Coan, J. A., Wagemans, F. M. A., Missler, M. A., Beest, I. van, Lindenberg, S., \& Tops, M. (2015). A theory of social thermoregulation in human primates. Frontiers in Psychology, 6.

Jänig, W. (2018). Peripheral thermoreceptors in innocuous temperature detection. In Handbook of clinical neurology (Vol. 156, pp. 47-56). Elsevier.

Jensen, K. B., Regenbogen, C., Ohse, M. C., Frasnelli, J., Freiherr, J., \& Lundström, J. N. (2016). Brain activations during pain: a neuroimaging meta-analysis of patients with pain and healthy controls. Pain, 157(6), 12791286.

Kastrati, G., Thompson, W. H., Schiffler, B., Fransson, P., \& Jensen, K. B. (2022). Brain Network Segregation and Integration during Painful Thermal Stimulation. Cerebral Cortex.

Kastrati, G., Rosén, J., Thompson, W. H., Chen, X., Larsson, H., Nichols, T. E., ... \& Jensen, K. B. (2022). Genetic Influence on Nociceptive Processing in the Human Brain-A Twin Study. Cerebral Cortex, 32(2), 266274.

Katkin, E. S., Reed, S. D., \& Deroo, C. (1983). A methodological analysis of 3 techniques for the assessment of individual-differences in heartbeat detection. Psychophysiology, Vol. 20, No. 4, pp. 452-452.

Ketai, L. H., Komesu, Y. M., Dodd, A. B., Rogers, R. G., Ling, J. M., \& Mayer, A. R. (2016). Urgency urinary incontinence and the interoceptive network: a functional magnetic resonance imaging study. American journal of obstetrics and gynecology, 215(4), 449-e1. 
Khalsa, S. S., Adolphs, R., Cameron, O. G., Critchley, H. D., Davenport, P. W., Feinstein, J. S., ... \& Zucker, N. (2018). Interoception and mental health: a roadmap. Biological Psychiatry: Cognitive Neuroscience and Neuroimaging, 3(6), 501-513.

Kirsch, L. P., Krahé, C., Blom, N., Crucianelli, L., Moro, V., Jenkinson, P. M., \& Fotopoulou, A. (2018). Reading the mind in the touch: Neurophysiological specificity in the communication of emotions by touch. Neuropsychologia, 116, 136-149.

Kirsch, L. P., Besharati, S., Papadaki, C., Crucianelli, L., Bertagnoli, S., Ward, N., ... \& Fotopoulou, A. (2020). Damage to the right insula disrupts the perception of affective touch. Elife, 9, e47895.

Kleckner, I. R., Zhang, J., Touroutoglou, A., Chanes, L., Xia, C., Simmons, W. K., ... \& Barrett, L. F. (2017). Evidence for a large-scale brain system supporting allostasis and interoception in humans. Nature human behaviour, 1(5), 1-14.

Knapp-Kline, K., Ring, C., Emmerich, D., \& Brener, J. (2021). The effects of vibrotactile masking on heartbeat detection: evidence that somatosensory mechanoreceptors transduce heartbeat sensations. Psychophysiology, 58(6), e13817.

Krahé, C., Springer, A., Weinman, J. A., \& Fotopoulou, A. K. (2013). The social modulation of pain: others as predictive signals of salience-a systematic review. Frontiers in human neuroscience, 7, 386.

Kress, I. U., Minati, L., Ferraro, S., \& Critchley, H. D. (2011). Direct skin-to-skin vs. indirect touch modulates neural responses to stroking vs. tapping. Neuroreport, 22(13), 646.

Kulkarni, B., Bentley, D. E., Elliott, R., Youell, P., Watson, A., Derbyshire, S. W. G., ... \& Jones, A. K. P. (2005). Attention to pain localization and unpleasantness discriminates the functions of the medial and lateral pain systems. European Journal of Neuroscience, 21(11), 3133-3142.

Kurz, A. (2008). Physiology of thermoregulation. Best Practice \& Research Clinical Anaesthesiology, 22(4), 627644.

Kwan, C. L., Crawley, A. P., Mikulis, D. J., \& Davis, K. D. (2000). An fMRI study of the anterior cingulate cortex and surrounding medial wall activations evoked by noxious cutaneous heat and cold stimuli. Pain, 85(3), 359-374.

Larsson, D. E., Esposito, G., Critchley, H. D., Dienes, Z., \& Garfinkel, S. N. (2021). Sensitivity to changes in rate of heartbeats as a measure of interoceptive ability. Journal of Neurophysiology, 126(5), 1799-1813.

Legrand, N., Nikolova, N., Correa, C., Brændholt, M., Stuckert, A., Kildahl, N., ... \& Allen, M. (2021). The heart rate discrimination task: a psychophysical method to estimate the accuracy and precision of interoceptive beliefs. Biological Psychology, 108239.

Lloyd, D. M., Gillis, V., Lewis, E., \& Farrell, M. J. (2013). Pleasant touch moderates the subjective but not objective aspects of body perception. Frontiers in behavioral neuroscience, 7, 207.

Löken, L. S., Wessberg, J., McGlone, F., \& Olausson, H. (2009). Coding of pleasant touch by unmyelinated afferents in humans. Nature neuroscience, 12(5), 547.

Montague, A. (1971). Touching: The Human Significance of the Skin (Columbia UP).

Morrison, I., Löken, L. S., \& Olausson, H. (2010). The skin as a social organ. Experimental Brain Research, 204(3), 305-314.

Morrison, I., Perini, I., \& Dunham, J. (2013). Facets and mechanisms of adaptive pain behavior: predictive regulation and action. Frontiers in human neuroscience, 7, 755.

Morrison, I. (2016). Keep calm and cuddle on: social touch as a stress buffer. Adaptive Human Behavior and Physiology, 2, 344-362.

Morrison, I. (2016a). ALE meta-analysis reveals dissociable networks for affective and discriminative aspects of touch. Human Brain Mapping, 37, 1308-1320.

Morrison, I. (2016b). CT afferent-mediated affective touch: brain networks and functional hypotheses. In $\mathrm{H}$. Olausson, I. Morrison, J. Wessberg, F. McGlone (Eds.), Affective touch and the neurophysiology of CT afferents. Amsterdam: Springer

Mower, G. D. (1976). Perceived intensity of peripheral thermal stimuli is independent of internal body temperature. Journal of comparative and physiological psychology, 90(12), 1152.

Murphy, J., Brewer, R., Coll, M. P., Plans, D., Hall, M., Shiu, S. S., ... \& Bird, G. (2019). I feel it in my finger: Measurement device affects cardiac interoceptive accuracy. Biological psychology, 148, 107765.

Muzik, O., \& Diwadkar, V. A. (2016). In vivo correlates of thermoregulatory defense in humans: Temporal course of sub-cortical and cortical responses assessed with fMRI. Human brain mapping, 37(9), 3188-3202. 
Muzik, O., Baajour, S., Bressler, S., \& Diwadkar, V. A. (2020). Directional interactions between constituents of the human large-scale thermoregulatory network. Brain Topography, 33, 489-503.

Nakamura, K., \& Morrison, S. F. (2008). A thermosensory pathway that controls body temperature. Nature neuroscience, 11(1), 62-71.

Oi, H., Hashimoto, T., Nozawa, T., Kanno, A., Kawata, N., Hirano, K., ... \& Kawashima, R. (2017). Neural correlates of ambient thermal sensation: an fMRI study. Scientific reports, 7(1), 1-11.

Olausson, H., Lamarre, Y., Backlund, H., Morin, C., Wallin, B. G., Starck, G., ... \& Bushnell, M. C. (2002). Unmyelinated tactile afferents signal touch and project to insular cortex. Nature neuroscience, 5(9), 900904.

Olausson, H., Wessberg, J., McGlone, F., \& Vallbo, Å. (2010). The neurophysiology of unmyelinated tactile afferents. Neuroscience \& Biobehavioral Reviews, 34(2), 185-191.

Park, H. D., \& Blanke, O. (2019). Coupling inner and outer body for self-consciousness. Trends in cognitive sciences, 23(5), 377-388

Pawling, R., Cannon, P. R., McGlone, F. P., \& Walker, S. C. (2017). C-tactile afferent stimulating touch carries a positive affective value. PloS one, 12(3), e0173457.

Perini, I., Bergstrand, S., and Morrison, I. (2013). Where pain meets action in the human brain. Journal of Neuroscience. 33, 15930-15939

Plans, D., Ponzo, S., Morelli, D., Cairo, M., Ring, C., Keating, C. T., ... \& Bird, G. (2021). Measuring interoception: The phase adjustment task. Biological Psychology, 165, 108171.

Proffitt, D. R. (2006). Embodied Perception and the Economy of Action. Perspectives on Psychological Science, 1(2), 110-122.

Proske, U., \& Gandevia, S. C. (2012). The proprioceptive senses: their roles in signaling body shape, body position and movement, and muscle force. Physiological reviews.

Quigley, K. S., Kanoski, S., Grill, W. M., Barrett, L. F., \& Tsakiris, M. (2021). Functions of interoception: from energy regulation to experience of the self. Trends in Neurosciences, 44(1), 29-38.

Radziun, D., Crucianelli, L., \& Ehrsson, H. H. (2021). Limits of cross-modal plasticity? Short-term visual deprivation does not enhance cardiac interoception, thermosensation, or tactile spatial acuity. Biological Psychology, 108248.

Rainville, P., Duncan, G. H., Price, D. D., Carrier, B., \& Bushnell, M. C. (1997). Pain affect encoded in human anterior cingulate but not somatosensory cortex. Science, 277(5328), 968-971.

Ring, C., \& Brener, J. (1996). Influence of beliefs about heart rate and actual heart rate on heartbeat counting. Psychophysiology, 33(5), 541-546.

Ring, C., Brener, J., Knapp, K., \& Mailloux, J. (2015). Effects of heartbeat feedback on beliefs about heart rate and heartbeat counting: A cautionary tale about interoceptive awareness. Biological psychology, 104, 193198.

Ring, C., \& Brener, J. (2018). Heartbeat counting is unrelated to heartbeat detection: A comparison of methods to quantify interoception. Psychophysiology, 55(9), e13084.

Rolls, E. T. (2010). The affective and cognitive processing of touch, oral texture, and temperature in the brain. Neuroscience \& Biobehavioral Reviews, 34(2), 237-245.

Rolls, E. T., O’Doherty, J., Kringelbach, M. L., Francis, S., Bowtell, R., \& McGlone, F. (2003). Representations of pleasant and painful touch in the human orbitofrontal and cingulate cortices. Cerebral cortex, 13(3), 308317.

Ross, A., \& Brener, J. (1981). Two procedures for training cardiac discrimination: A comparison of solution strategies and their relationship to heart rate control. Psychophysiology, 18(1), 62-70.

Salvato, G., Richter, F., Sedeño, L., Bottini, G., \& Paulesu, E. (2020). Building the bodily self-awareness: Evidence for the convergence between interoceptive and exteroceptive information in a multilevel kernel density analysis study. Human brain mapping, 41(2), 401-418.

Satinoff, E. (1978). Neural organization and evolution of thermal regulation in mammals. Science, 201(4350), 1622.

Schandry, R. (1981). Heart beat Perception and Emotional Experience. Psychophysiology 18 (4), 483-488.

Serino, A., \& Haggard, P. (2010). Touch and the body. Neuroscience \& Biobehavioral Reviews, 34(2), $224-236$.

Seth, A. K. (2013). Interoceptive inference, emotion, and the embodied self. Trends in cognitive sciences, 17(11), 565-573. 
Sherrington, C. S. (1906). The Integrative Action of the Nervous System. New Haven, CT: Yale University Press Sherrington, C.S. (1948). The integrative action of the nervous system. Cambridge, UK: Cambridge University Press.

Sherwood, S. C., \& Huber, M. (2010). An adaptability limit to climate change due to heat stress. Proceedings of the National Academy of Sciences, 107(21), 9552-9555.

Simmons, W. K., Avery, J. A., Barcalow, J. C., Bodurka, J., Drevets, W. C., \& Bellgowan, P. (2013). Keeping the body in mind: insula functional organization and functional connectivity integrate interoceptive, exteroceptive, and emotional awareness. Human brain mapping, 34(11), 2944-2958.

Solinski, H. J., \& Hoon, M. A. (2019). Cells and circuits for thermosensation in mammals. Neuroscience letters, 690, 167-170.

Strigo, I. A., Matthews, S. C., \& Simmons, A. N. (2010). Right anterior insula hypoactivity during anticipation of homeostatic shifts in major depressive disorder. Psychosomatic medicine, 72(3), 316.

Strigo, I. A., \& Craig, A. D. (2016). Interoception, homeostatic emotions and sympathovagal balance. Philosophical Transactions of the Royal Society B: Biological Sciences, 371(1708), 20160010.

Tansey, E. A., \& Johnson, C. D. (2015). Recent advances in thermoregulation. Advances in physiology education.

Terrien, J., Perret, M., \& Aujard, F. (2011). Behavioral thermoregulation in mammals: a review. Frontiers in Bioscience, 16(4), 1428-1444.

Tsakiris, M. (2017). The multisensory basis of the self: from body to identity to others. The Quarterly Journal of Experimental Psychology, 70(4), 597-609.

Vallbo, Å. B., Olausson, H., \& Wessberg, J. (1999). Unmyelinated afferents constitute a second system coding tactile stimuli of the human hairy skin. Journal of neurophysiology, 81(6), 2753-2763.

van Stralen, H. E., van Zandvoort, M. J., Hoppenbrouwers, S. S., Vissers, L. M., Kappelle, L. J., \& Dijkerman, H. C. (2014). Affective touch modulates the rubber hand illusion. Cognition, 131(1), 147-158.

von Mohr, M., \& Fotopoulou, A. (2018). The Cutaneous borders of interoception: Active and social inference of pain and pleasure on the skin. In M. Tsakiris \& H. de Preester (Eds.), The Interoceptive Mind: From Homeostasis to Awareness (1st ed., pp. 102-120). Oxford, United Kingdom: Oxford University Press.

Walker, S. C., \& McGlone, F. P. (2013). The social brain: neurobiological basis of affiliative behaviours and psychological well-being. Neuropeptides, 47(6), 379-393.

Wessberg, J., Olausson, H., Fernström, K. W., \& Vallbo, Å. B. (2003). Receptive field properties of unmyelinated tactile afferents in the human skin. Journal of neurophysiology, 89(3), 1567-1575.

Whitehead, W. E., Drescher, V. M., Heiman, P., \& Blackwell, B. (1977). Relation of heart rate control to heartbeat perception. Biofeedback and Self-regulation, 2(4), 371-392.

Whitehead, W. E., \& Dreschner, V. M. (1980). Perception of Gastric Contractions and Self-Control of Gastric motility. Psychophysiology, 17(6), 552-558

Zaki, J., Davis, J. I., \& Ochsner, K. N. (2012). Overlapping activity in anterior insula during interoception and emotional experience. Neuroimage, 62(1), 493-499.

Zamariola, G., Maurage, P., Luminet, O., \& Corneille, O. (2018). Interoceptive accuracy scores from the heartbeat counting task are problematic: Evidence from simple bivariate correlations. Biological Psychology, 137, $12-17$

Zimprich, D., Nusser, L., \& Pollatos, O. (2020). Are interoceptive accuracy scores from the heartbeat counting task problematic? A comment on Zamariola et al. (2018). Biological Psychology, 152, 107868. 